\title{
Mathematical Modeling for Quantum Electron Wave Therapy
}

\author{
Gianamar Giovannetti-Singh ${ }^{1,2},{\text { Sixian } \text { Zhao }^{2}}^{2}$ \\ ${ }^{1}$ Department of Physical Sciences, Open University, Cambridge, UK \\ ${ }^{2}$ Science Department, Parkside Federation Academy, Cambridge, UK \\ Email: gianamar@ieee.org
}

Received December 30, 2011; revised February 5, 2012; accepted February 15, 2012

\begin{abstract}
The hypothesis suggesting that the physical process of quantum tunneling can be used as a form of cancer therapy in electron ionization radiotherapy was suggested in the IEEE International Conference on Electric Information and Control Engineering by G. Giovannetti-Singh (2012) [1]. The hypothesis used quantum wave functions and probability amplitudes to find probabilities of electrons tunneling into a cancer cell. In addition, the paper explained the feasibilities of the therapy, with the use of nanomagnets. In this paper, we calculate accurate probability densities for the electron beams to tunnel into cancer cells. We present our results of mathematical modeling based on the helical electron wave function, which "tunnel" into a cancer cell, therefore ionizing it more effectively than in conventional forms of radiotherapy. We discuss the advantages of the therapy, and we explain how quantum mechanics can be used to create new cancer therapies, in particular our suggested Quantum Electron Wave Therapy.
\end{abstract}

Keywords: Electron Wave Therapy; Quantum Tunneling; Wave Function; Quantum Theory; Cancer Therapy

\section{Introduction}

This paper builds on an earlier model suggesting that quantum tunneling techniques which, in theory can be used successfully in cancer therapy [1]. This approach used the wave function of an electron to calculate the energy needed for an electron to "tunnel" through the cancerous membrane of the cell. It was suggested that this method could be more effective than conventional radiotherapy in treating cancer for the following reasons:

- In addition, the electron beam (which tunnels) can travel directly to the nuclei of the cancer cells.

- It is more direct, as instead of harming the surrounding cells it only targets the cancer cells through careful instrumentation and accuracy.

- According to the laws of momentum, there will be no impact from the electrons as they aim into the cells' nuclei because if they tunnel, they must be travelling as a wave.

Nevertheless, [1] only suggests a hypothesis, however in this paper; we calculate the probability density of a single electron (in one dimension) tunneling into a cancer cell.

In this paper, we give accurately calculated figures of the probability density from the one-dimensional, time independent electron wave function, and we discuss the graph describing the electrons' helical motion.

\section{Quantum Wave Functions}

Using the three parametric functions below, we combine them to create a helical graph which denotes the wave function, and hence the trail of an electron through space as a wave.

$$
\begin{gathered}
x=M \cos (\phi) \\
y=M \sin (\phi) \\
z=\phi
\end{gathered}
$$

where $x, y$ and $z$ are the three Cartesian axes, $M$ is the modulus of the helix, also equal to the amplitude of the helical wave. The parameter $M$ is introduced to differentiate between wave functions of helixes of different amplitudes. $\phi$ is the phase of the wave.

Given that:

$$
\begin{aligned}
\psi(x) & =M e^{i x} \because M e^{i \phi} \\
& =M \cos (\phi)+i M \sin (\phi)
\end{aligned}
$$

[2], we produce a graph of the parametric Equations (1)(3).

In this case, the spiral will be no more than five times the diameter of the electron, therefore we can assume that $M=5 \times 10^{-15} \mathrm{~m}$. Using this figure, we can make calculations for the electron gun model [3]. 


\section{Mathematical Modeling}

\subsection{Quantum Physical Properties of the Electron Wave}

The process of mathematically modeling the electron wave function will find the realistic most efficient distance from the tumor by carrying out a triple integration of the wave function through a cancer cell (assuming that a cell is mostly $\mathrm{H}_{2} \mathrm{O}$ ), to find a probability density of the electron tunneling into the cell [4].

We calculate the minimum and maximum energy required for the electron to be in waveform from Planck's equations for the energy of a wave; $E=h v$ [5], we calculate the minimum energy, which is determined by Equation (3) because of $\phi$, the phase of the helix.

This equation leaves us with the simple relation between the frequency $(f)$ and the phase $(\phi)$. As the coefficient of $\phi$ increases, the denominator of $f$ divides by two.

$$
\left(v=\frac{1}{8} \mid z=\phi\right)
$$

However, the frequency of the wave is a limit because although it will continue to approach zero, it will never reach, therefore, the definitions between particle and wave becomes fuzzy.

JB Hagen (2009) [6] suggested a different model for phase-angular frequency relation in electromagnetic waves; however, according to the laws of quantum mechanics, this is equivalent for electrons [7]. This model states that

$$
\begin{aligned}
\phi(t) & =\int \omega(t) \mathrm{d} t=\int\left[\omega_{0}+k_{0} A \cos \left(\omega_{a} t\right)\right] \\
& =\omega_{0} t+\frac{k_{0} A}{\omega_{a}} \sin \sin \left(\omega_{a} t\right)+\phi_{0}
\end{aligned}
$$

We now substitute into Planck's equation for the energy of a wave, and we obtain the values:

$$
E=\frac{1}{8} h=8.28258696 \times 10^{-35} \mathrm{~J}
$$

This is the minimum energy required for an electron to tunnel into the cancer cell. In addition, we can calculate the maximum energy that the electron can contain to be a wave.

As the electron wave-function verges towards the limit of a straight line rather than the helix graph, the behavior of the electron begins to be more particle-like, as it no longer can pass through "barriers", and must give in to the laws of momentum. Einstein's equation for the momentum of particles is the following: $E^{2}=m^{2} c^{4}+p^{2} c^{2}[8]$.

Therefore, we can calculate the electron's momentum of impact on the cell wall. Given that the equation for kinetic energy is $E=\frac{1}{2} m v^{2}$, we isolate $v$ to then calcu-

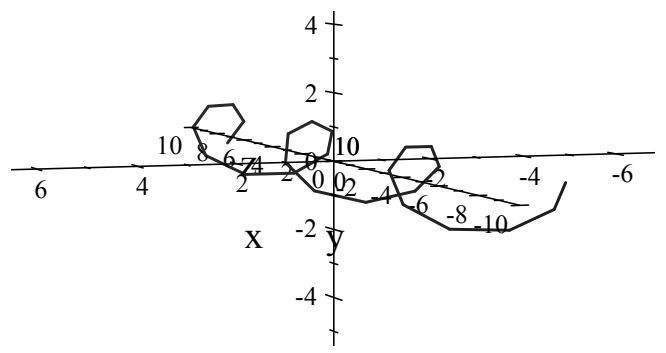

Figure 1. Electron wave function helical graph for parametric wave equations $x=M \cos (\phi), y=M \sin (\phi), z=\phi$.

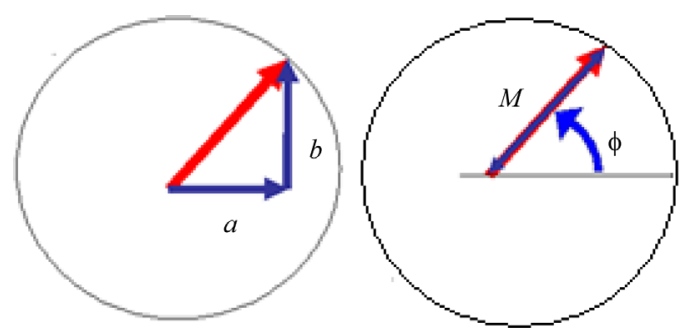

Figure 2. Cross-section view of the helix [2].

late Einstein's equation for the momentum of a particle.

$$
\begin{gathered}
v=\sqrt{\frac{2 E}{m}} \\
v=\sqrt{\frac{2\left(8.28258696 \times 10^{-35} \mathrm{~J}\right)}{9.10938188 \times 10^{-31} \mathrm{~kg}}} \\
=0.0134850805746 \mathrm{~m} \cdot \mathrm{s}^{-1}
\end{gathered}
$$

Therefore, by substitution, we calculate the velocity of the electron as $0.0134850805746 \mathrm{~ms}^{-1}$. Now we can calculate the momentum of the electron to be:

$$
\begin{aligned}
& 9.10938188 \times 10^{-31} \mathrm{~kg} \cdot 0.0134850805746 \mathrm{~m} \cdot \mathrm{s}^{-1} \\
& =1.22840743 \times 10^{-32} \mathrm{~N} \cdot \mathrm{s}
\end{aligned}
$$

\subsection{Probability Density}

We calculate a one-dimensional probability density of an electron appearing "past the barrier" into the cancer cell. We obtain this figure from the one-dimensional wave function: $\psi(x)=M e^{i x}{ }^{1}$ [2], which is an exponential function. We can therefore plot a 2D graph which represents our probability density function [9].

The integral which calculates the probability density function is calculated between two points, in this case, the diameter of a cancer cel1 [10].

$$
P_{b<x<a}=\int_{a}^{b}|\psi(x)|^{2} \mathrm{~d} x
$$

In this example, the integral would be:

$$
P_{10^{-8}<x<0}=\int_{0}^{10^{-8}}|\psi(x)|^{2} \mathrm{~d} x
$$

We calculate the integral from $\psi(x)=M e^{i x} . M$ is the

${ }^{1}$ The $3 \mathrm{D}$, time-dependent wave function for an electron is $\psi(r, t)=$ $A e^{i(k \cdot r-\omega t)}$. 


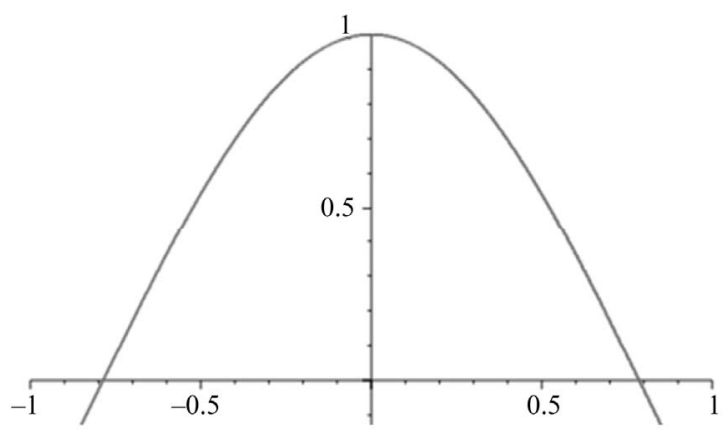

Figure 3. One-dimensional wave function graph for $\psi(x)$.

modulus, the radius from the centre of the corkscrew to the limit $5 \times 10^{-15} \mathrm{~m}$.

We first normalize the equation into the form of a definite integral between $\infty$ and $-\infty$.

$$
P_{\infty<x<-\infty}=\int_{-\infty}^{\infty}|\psi(x)|^{2} \mathrm{~d} x=1
$$

We now obtain a numerical value for the probability density distribution:

$$
P_{10^{-8}<x<0}=\int_{0}^{10^{-8}}\left|M e^{i x}\right|^{2} \mathrm{~d} x=5 \times 10^{-37}
$$

\section{Conclusion}

We conclude this paper by providing numerical values for a probability density of a single electron tunneling into a cancer cell. In addition, we multiply this value by the number of electrons that we will shoot into a cancer cell, therefore increasing the electrons' probabilities of tunneling. Given that in an electron beam with the width of $1 \mathrm{~cm}$, there can be $5 \times 10^{13}$ electrons, and our beam can be up to $10 \mathrm{~cm}$ long, we therefore calculate the volume of the beam (approximating a cubic prism) as being $10 \mathrm{~cm}^{3}$. In addition, we approximate that $1.25 \times 10^{48}$ electrons can be within the space. According to the probability density which we have obtained, if we multiply our value of $5 \times 10^{-37}$ by $10^{36}$ (the maximum number of electrons which we want to use) we obtain a probability of 0.5 , which is a figure with a reasonable probability to allow electron wave therapy to commence.

\section{Acknowledgements}

We would like to thank Hannah Jones, Adam Biltcliffe and Professor Emanuele Giovannetti for constant support and fruitful discussions on the research.

\section{REFERENCES}

[1] G. Giovannetti-Singh, "Electron Ionisation Therapy via Quantum Tunnelling," Proceedings of the 2nd International Conference on Electric Information and Control Engineering, Lushan, 6-8 April 2012.

[2] J. Rodenburg, "The Value of the Electron Wave," Rodenburg, 2010. http://www.rodenburg.org/theory/y100.html

[3] T. Okubo, Y. Kato and G. Matsuaka, "Method and System for Measuring the Diameter of an Electron Beam," United States Patent No. 4336597, 1980.

[4] W. D. Donachie, "Relationship between Cell Size and Time of Initiation of DNA Replication," Nature, Vol. 219, No. 5158, 1968, pp. 1077-1079. doi:10.1038/2191077a0

[5] M. Planck, "Quantum Theory," Annalen der Physik, WileyVCH Verlag GmbH \& Co. KgaA, Berlin, 1901.

[6] J. B. Hagen, "Radio-Frequency Electronics: Circuits and Applications," Cambridge University Press, Cambridge, 2009, pp. 291-294.

[7] A. Einstein, "The Photoelectric Effect," Annalen der Physik, Wiley-VCH Verlag GmbH \& Co. KgaA, Berlin, 1905.

[8] A. Einstein, "On the Electrodynamics of Moving Bodies," Annalen der Physik, Wiley-VCH Verlag GmbH \& Co. KgaA, Berlin, 1905.

[9] H. Jeffreys, "Theory of Probability," 2nd Edition, Oxford University Press, Oxford, 1948.

[10] E. Richtig, G. Langmann, K. Müllner, J. Smolle, "Calculated Tumour Volume as a Prognostic Parameter for Survival in Choroidal Melanomas" Eye, Vol. 18, No. 6, pp. 619-623. 2004. doi:10.1038/sj.eye. 6700720 